\title{
Observations of the companion to the pulsar PSR B1718-19 $\star \star \star \star$
}

\section{The role of tidal circularisation}

\author{
T. Janssen ${ }^{1}$ and M. H. van Kerkwijk ${ }^{1,2}$ \\ 1 Astronomical Institute, Utrecht University, PO Box 80000, 3508 TA Utrecht, The Netherlands \\ e-mail: T.Janssen@phys .uu.nl \\ 2 Department of Astronomy and Astrophysics, University of Toronto, 60 St George Street, Toronto, Ontario M5S 3H8, Canada \\ e-mail: mhvk@astro.utoronto.ca
}

Received 3 January 2005 / Accepted 6 April 2005

\begin{abstract}
We present optical and infrared observations taken with the Very Large Telescope of the eclipsing binary pulsar system PSR B1718-19. The candidate companion of the pulsar, identified earlier in Hubble Space Telescope observations, has been detected in all three bands, $R, I$, and $J$. These detections allowed us to derive constraints on temperature, radius, and mass, pointing to a companion that has expanded to a radius between one of a main sequence star and one at the Roche-limit. We focus on the role of tidal circularisation in the system, which will have transformed the initially eccentric orbit expected from formation scenarios into the nearly circular orbit presently observed. Based on simple energy balance arguments, we are able to draw a picture of the companion's evolution resulting from the energy deposition in the star due to circularisation. In this picture, our measurement of the companion's parameters is consistent with the expected initial eccentricity. However, with the present understanding of tidal dissipation, it remains difficult to account for the short time in which the system was circularised.
\end{abstract}

Key words. binaries: eclipsing - pulsars: individual: PSR B1718-19 - stellar dynamics

\section{Introduction}

The binary radio pulsar PSR B1718-19 has a 1-s spin period and was discovered by Lyne et al. (1993) in the direction of globular cluster NGC 6342. The pulsar is young and has a high magnetic field: from the pulse period and its derivative, one infers $\tau_{\text {char }}=P /(2 \dot{P})=10 \mathrm{Myr}$ and $B=1.5 \times 10^{12} \mathrm{G}$. The binary orbit is circular, has a period of $6.2 \mathrm{~h}$, and is accompanied by eclipses at low radio frequencies, of $400-600 \mathrm{MHz}$. The eclipsing material is probably the companion's wind.

The formation scenarios proposed (Lyne et al. 1993; Ergma 1993; Wijers \& Paczynski 1993; Zwitter 1993; Burderi \& King 1994; Ergma et al. 1996) fall into two main categories: (i) partial recycling of an old neutron star in a close encounter with other stars in the cluster core, and (ii) accretion-induced collapse (AIC) of a white dwarf. Van Kerkwijk et al. (2000) detected a candidate for the pulsar companion. The object's faintness was a strong indication of scenario (i), though scenario (ii) could not be excluded definitively.

Wijers \& Paczynski (1993) noted that either formation scenario leads to an eccentric binary orbit, and tidal circularisation

$\star$ Based on observations collected at the European Southern Observatory, Chile (ESO Programme 67.D-0058).

$\star \star$ Full Table 2 is only available in electronic form at the CDS via anonymous ftp to cdsarc.u-strasbg.fr $(130.79 .128 .5)$ or via http://cdsweb.u-strasbg.fr/cgi-bin/qcat?J/A+A/439/433 must have worked to reduce the eccentricity to the currently observed low value $(e \lesssim 0.005)$. Thus, PSR B1718-19 might serve as an observational constraint on circularisation theory in a parameter region different from the region covered by constraints that are currently available: tidal cutoff periods of solartype binaries (see, e.g., Mathieu et al. 2004, and references therein), binaries containing a giant star (Verbunt \& Phinney 1995), or planets (Wu 2003).

Circularisation efficiency depends strongly on the object's radius, but this is poorly constrained by the single-band detection of Van Kerkwijk et al. (2000). This motivated us to collect further observations to obtain a direct measurement of temperature and radius. In this paper, we describe the results. In Sect. 2, we describe the reduction and show our results in colour-colour and colour-magnitude diagrammes. We use these in Sect. 3 to put further constraints on the mass, radius, and temperature of the companion, and in Sect. 4 we investigate the implied role of tidal circularisation, focusing on the circularisation timescale and the consequences of tidal dissipation in the companion.

\section{Observations}

The observations on which we report here were done for us in Service Mode in June 2001 with the Very Large Telescope (VLT) at ESO (Paranal) through filters $R, I$, and $J$ 
Table 1. Log of observations.

\begin{tabular}{cccccccc}
\hline \hline Instrument & Band & Night & $t_{\text {int }}(\mathrm{s})$ & Seeing $\left({ }^{\prime \prime}\right)$ & \# EXP & STD-field ${ }^{a}$ & $\Delta \sec z^{b}$ \\
\hline \multirow{2}{*}{ VLT-FORS2 } & $I$ & 2001 Jun. 20 & 257 & $0.48-0.61$ & 8 & PG1323 & 0.04 \\
& & 2001 Jun. 22 & 240,257 & $0.44-0.67$ & 8 & SA92-249 & 0.00 \\
VLT-FORS2 & $R$ & 2001 Jun. 20 & 317 & $0.51-0.68 / 0.75^{c}$ & $11 / 12^{c}$ & PG1323 & 0.02 \\
& & 2001 Jun. 22 & 300,317 & $0.48-0.66 / 0.71-0.83^{c}$ & $11 / 13^{c}$ & SA92-249 & 0.02 \\
VLT-ISAAC & $J$ & 2001 Jun. 7 & 30.30 & $\ldots / 0.59-0.87^{c}$ & $0 / 16^{c}$ & S860-D & 0.00 \\
& & 2001 Jun. 14 & 30.30 & $0.36-0.56$ & 48 & S860-D & 0.07 \\
HST-WFPC2 & $F 702 W$ & 1997 Mar. 8 & 700 & 0.046 & 12 & $\ldots$ & $\ldots$ \\
\hline
\end{tabular}

\footnotetext{
$a$ The standard magnitudes are taken from Stetson (2000) for $I$ and $R$ and from Persson et al. (1998) for $J$.

${ }^{b}$ This column lists the difference in airmass between the single exposure science frame that was used for calibration and the standard frame (see text).

${ }^{c}$ The frames were selected on seeing value. Listed in the seeing column is, before the slash, the seeing range of frames included in the mosaic image, and, after the slash, the seeing range of frames that were excluded. In the \# EXP column the number of exposures included in the mosaic image is displayed with, after the slash, the total number of exposures taken.
}

(see Table 1). In this section we will describe their reduction and photometry.

We will also use the data presented in Van Kerkwijk et al. (2000): images obtained through the $F 702 W$ filter with the Wide Field and Planetary Camera 2 (WFPC2) of the Hubble Space Telescope (HST) in March 1997. Only the data from the Planetary Camera $\left(800 \times 800\right.$ pixels, at $0{ }^{\prime} 046$ pixel $\left.^{-1}\right)$ will be used for the present paper. As will be discussed in Sect. 2.3, we were unable to derive reliable photometry from these data. The $F 702 \mathrm{~W}$ images still serve, however, for measurements of the proper motions of stars in the field. The astrometry done by Van Kerkwijk et al. (2000) enables us to directly identify the candidate for the pulsar's companion in our images, shown in Fig. 1.

\subsection{Infrared observations in $J$}

The $J$-band observations were done with the short-wavelength channel of the Infrared Spectrometer And Array Camera (ISAAC; Moorwood et al. 1998) mounted on the $8.2 \mathrm{~m}$ Antu (Unit Telescope 1). The detector was a Rockwell Hawaii 1024× 1024 pixel array with a plate scale of 0.148 pixel $^{-1}$.

The ISAAC-observations needed to be corrected for two instrumental effects: (i) the odd-even column effect, which can be seen as an offset between the odd and even columns of the array, and (ii) the electrical ghost, which consists of an additional signal proportional to the sum of the intensity along a given row and the row 512 rows away (see the ISAAC Data Reduction Guide ${ }^{1}$, Amico et al. 2001). We employ ESO's software package ECLIPSE (Devillard 1997) for these corrections. Next, we use MIDAS to subtract the dark current from all frames and divide them by twilight flat fields.

From the images, it was clear that the seeing in the first observing night was much worse than in the second night (see

\footnotetext{
1 Available at http://www .eso.org/instruments/isaac/drg/ drg.ps
}

Table 1). We decided to exclude all 16 frames taken on the first night. We register the 48 frames of the second night with respect to each other, applying offsets rounded to the nearest integer pixel, and compute an average image. Bad pixels are excluded from this average. We regard a pixel as bad, if (i) it is located at rows 513 or 514 (2048 pixels), where bias-level variations with time prevent proper bias subtraction; (ii) the difference between a long and a short dark-current exposure at that location lies more than $7.5 \sigma$ away from the mean (57 pixels); (iii) it has a lower value than $2.75 \sigma$ below background in more than $50 \%$ of the frames (172 pixels), or (iv) if it is a hot pixel (59 pixels). Finally, in order to prevent problems with differences in the exposure time and distortion near the edges, we extract the central $600 \times 600$ pixel $\left(89 \times 89^{\prime \prime}\right)$ part of our mosaic image. In this subimage, the point-spread function (PSF) shows negligible variation.

We perform PSF-fitting photometry using the DAOPHOT II package (Stetson 1987). Determination of the PSF is hampered by the crowdedness of the field: one object per $4 \operatorname{arcsec}^{2}$, at a full width at half maximum ( $F W H M$ ) of 0.5 . We deal with this, following the suggestions of Stetson (1987), by first estimating the PSF based on several relatively isolated stars and then using this PSF to subtract neighbouring stars. A better PSF can be determined on the new image. This procedure has been repeated four times. With the resulting final PSF, the magnitudes of all stars are determined.

Observations of a standard star (Table 1) serve to calibrate the photometry. As these observations are taken with very short exposure times, the PSF varies greatly over the field. Hence, PSF-fitting is not reliable and we resort to aperture photometry. The standard star's aperture magnitudes can then be related to magnitudes of stars in a single exposure science frame, measured with a corresponding aperture. For this purpose, we select a science frame taken at airmass as close as possible to that at which the standard star was taken (see Table 1), so that there is no need to correct for atmospheric extinction. To account for 


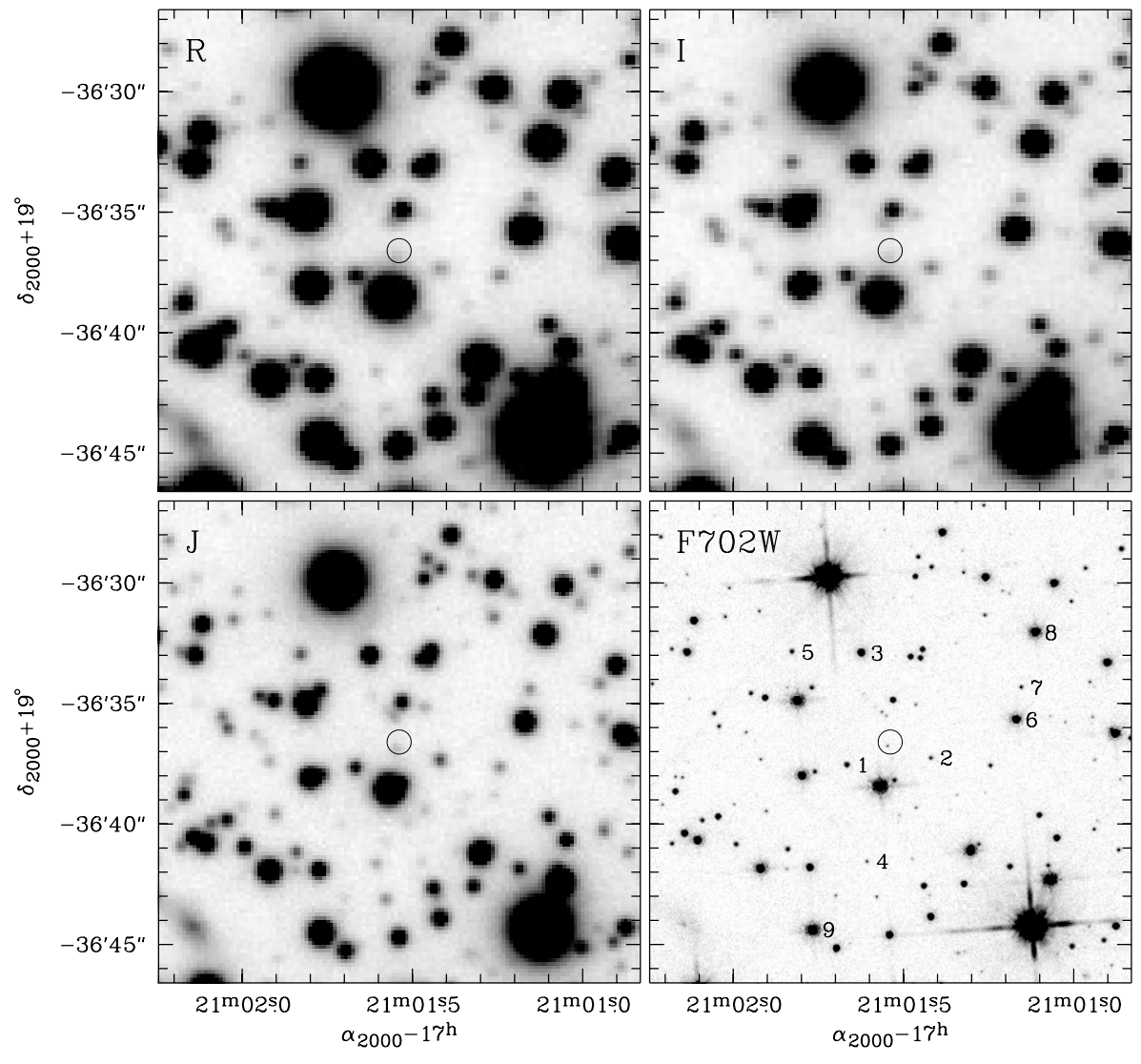

Fig. 1. Images of the field of PSR B1718-19. Shown are - clockwise the images in $R$ and $I$ (taken with VLTFORS2), in $F 702 W$ (HST-PC), and in $J$ (VLT-ISAAC). The $F 702 W$-observations have been presented in Van Kerkwijk et al. (2000), who also performed the astrometry to derive the $95 \%$ confidence error circle of radius 0.5 , centered on the position of the pulsar. This error circle is displayed in the images, and contains a detection of the candidate counterpart in all bands. For stars indicated by numbers in the F702W-panel, and for the PSR-companion we list positions and magnitudes in Table 2 .
Table 2. Positions and apparent magnitudes of the candidate companion of PSR B1718-19 and surrounding stars. The uncertainty in the photometric zero point is $0.02 \mathrm{mag}$ for $J$ and $<0.03 \mathrm{mag}$ in $R$ and $I$. The uncertainty in the astrometric tie is 0.06 in each coordinate (Van Kerkwijk et al. 2000). Photometry for all stars in the field, including uncertainties, is available at the CDS.

\begin{tabular}{|c|c|c|c|c|c|}
\hline ID & $\begin{array}{c}\alpha_{2000} \\
17^{\mathrm{h}} 21^{\mathrm{m}}\end{array}$ & $\begin{array}{c}\delta_{2000} \\
-19^{\circ} 36^{\prime}\end{array}$ & $R$ & $I$ & $J$ \\
\hline \multirow[t]{2}{*}{ PSR } & $01^{\mathrm{s}} .548$ & $36^{\prime \prime} 77$ & 25.87 & 24.25 & 22.59 \\
\hline & $\pm 0^{\varsigma} .004$ & \pm 0.06 & \pm 0.20 & \pm 0.08 & \pm 0.08 \\
\hline 1 & $01^{\mathrm{s}} .667$ & $37 . \prime 55$ & 23.31 & 22.09 & 20.56 \\
\hline 2 & $01^{\mathrm{s}} .421$ & $37^{\prime \prime} .26$ & 24.79 & 22.98 & 21.08 \\
\hline 3 & $01^{\mathrm{s}} .625$ & 32.91 & 21.00 & 20.35 & 19.37 \\
\hline 4 & $01^{\mathrm{s}} .608$ & $41^{\prime \prime} 54$ & 25.74 & 23.96 & 22.03 \\
\hline 5 & $01^{\mathrm{s}} .828$ & $322^{\prime \prime} 87$ & 23.55 & 22.53 & 21.14 \\
\hline 6 & $01^{\mathrm{s}} .171$ & $35^{\prime \prime} 67$ & 20.50 & 19.82 & 18.75 \\
\hline 7 & $01^{\mathrm{s}} .156$ & $34^{\prime \prime} 34$ & 24.82 & 23.49 & 21.67 \\
\hline 8 & $01^{\mathrm{s}} .114$ & $322^{\prime \prime} 06$ & 20.02 & 19.40 & 18.49 \\
\hline 9 & $01^{\mathrm{s}} .767$ & $44^{\prime \prime} 39$ & 19.59 & 18.95 & 17.99 \\
\hline
\end{tabular}

the quite different seeing of the standard and the science exposures, we use apertures with an equal radius in units of the image $F W H M$, not pixels. We used the trend of magnitude as a function of aperture to verify that using an aperture of the same radius in $F W H M$ indeed results in a superior match of the fraction of the flux inside a given aperture in two different frames.

Next, we compute an aperture correction to relate the aperture magnitude in the single science frame to the magnitudes determined from PSF fitting in the mosaic image. Out of necessity, since we do not have another infrared colour, we neglect any colour terms. From the ISAAC Data Reduction Guide (Amico et al. 2001, Sect. 5.5.1), this appears reasonable: the $J-K$ colour term is expected to differ from 0 by less than 0.01 , while we estimate that, overall, the total error in the calibration is $\sim 0.02 \mathrm{mag}$.

\subsection{Optical observations in $R$ and I}

The $R$ and $I$-band observations were carried out with the FOcal Reducer/low dispersion Spectrograph (FORS2; Seifert et al. 2000) mounted on Yepun (VLT-UT4). FORS was used in Standard Resolution imaging mode, in which the plate scale on the $2048 \times 2048$ pixel SITe detector is 0.201 pixel $^{-1}$.

The reduction was done in a similar fashion as described above for the ISAAC-observations. First, all frames are biassubtracted and divided by twilight flat fields. We select frames with a seeing lower than 0.7 (see Table 1) and compute an average mosaic of the registered frames, excluding 1329 pixels in each image located on a bad column, as well as pixels that were hit by cosmic rays. From the mosaics, we extract the central $400 \times 400$ pixel $\left(80 \times 80^{\prime \prime}\right)$ parts, which are covered by all individual frames and which have negligible distortion. On these subimages the photometry is done using procedures 
described above for the ISAAC data. For the calibration, we determine a weighted average from both observing nights for the zero point and the color term. This leads to a total error in the calibration of $\lesssim 0.03 \mathrm{mag}$ in both bands.

\subsection{Problems with the F702W-observations}

We tried to obtain photometry from the HST $F 702 \mathrm{~W}$ observations, both using HSTPHOT (Dolphin 2000) and following the instructions in the HST WFPC2 data handbook (Baggett 2002). We failed to obtain numbers that we felt were sufficiently reliable, for two reasons. First, independent of the reduction method, we found that in colour-colour diagrammes (e.g., $m_{F 702 W}-I$ vs. $I-J$ ) the root-mean-square scatter for bright, blue stars $(I-J<1.05)$ was as large as $0.026 \mathrm{mag}$, while in a comparable plot for $R-I$ (see Fig. 2), it is only 0.008 mag. This implies the WFPC2 data had a base noise of $\sim 0.02$ to $0.03 \mathrm{mag}$, which we do not understand. From discussions with Drs Andrew Dolphin and Peter Stetson (2004, personal comm.), we learned that such scatter is normally present, but that it should average out when one uses multiple frames. In our case, however, it did not, possibly because our dither pattern used integer pixel offsets. We also confirm it is present in other comparisons between ground-based and WFPC 2 data, such as those presented in van Kerkwijk \& Kulkarni (2001); for that field, we find that ACS/HRC data show much less scatter and are completely consistent with the ground-based data.

The second and more severe problem is that the two reduction schemes lead to a zero point that differs by $0.1 \mathrm{mag}$. We are confident that this is not because we performed an inappropriate aperture correction, such as the 0.1 mag offset between the standard aperture of 0 '. 5 and "nominal infinity"; we do not find such differences for images in other filters that we analysed. Since we were unable to identify the flaw in the reduction, we conclude that we cannot rely on the magnitude derived for the pulsar companion, and excluded the $F 702 \mathrm{~W}$ photometry from our analysis. We note, however, that within a 0.1 mag uncertainty, the magnitude found is consistent with that expected from our $R$ and $I$-band detections.

\subsection{Photometry and proper motions}

We used our calibrated photometry (Table 2 and on-line material), to construct colour-colour and colour-magnitude diagrammes; see Fig. 2. In the colour-magnitude diagramme, we find a rather broad main sequence, which suggests that many of the stars do not belong to the cluster.

As the HST observations were taken 4.25 years prior to the VLT-observations, we can try to separate cluster stars from fore- and background objects using proper motions. For this purpose, we measured positions in the HST PC and our bestseeing VLT observation, that in the $J$ band. The result is shown in Fig. 3. One sees that especially among brighter stars, a clustering of stars associated with NGC 6342 is apparent. This is confirmed in Fig. 2, where we use filled symbols to indicate stars that are likely cluster members (those within the dashed circle in Fig. 3): the brighter ones, with $J \lesssim 21$, form a clear,

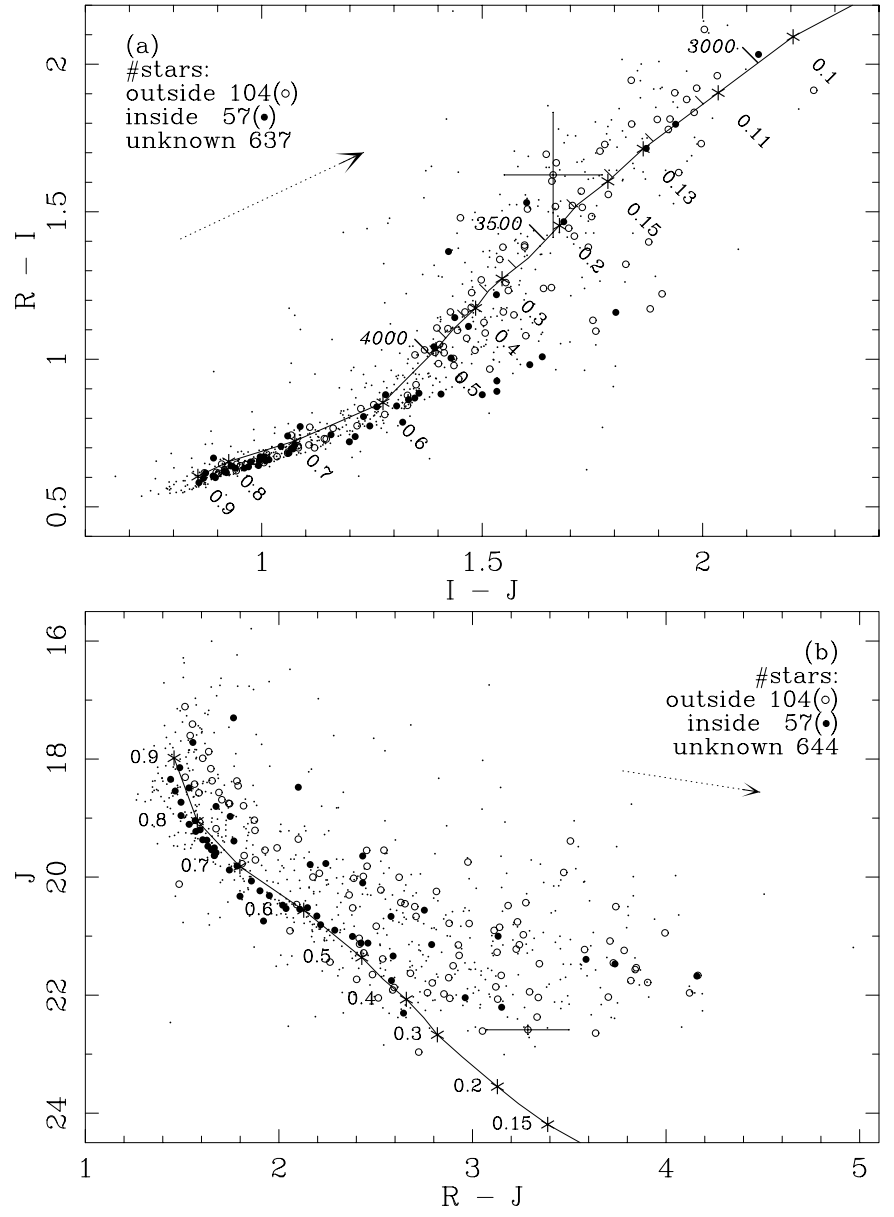

Fig. 2. a) Colour-colour and b) colour-magnitude diagrammes of the companion of PSR B1718-19 (error bars) and stars in the field. The filled circles denote stars with a proper motion inside the dashed circle of Fig. 3, which are likely to be associated with the cluster NGC 6342. The open circles indicate stars with a proper motion outside this circle. For stars denoted by a dot, no proper motion is available, since they were outside the field of view of the PC. The detection limit in $J$ is around $23 \mathrm{mag}$. The solid line represents model main sequence stars, labeled with their masses (in solar units), and - in italic typeface temperature (small tickmarks denote intervals of $100 \mathrm{~K}$ ). The models are corrected for a reddening $E_{V-I}=0.55$ and distance modulus $(m-M)_{0}=14.69$ (see Sect. 3). The dotted arrow denotes a reddening of $E_{V-I}=0.55 \pm 0.07$ (with the size of the solid part equal to the uncertainty).

narrow sequence that is well matched by the expected main sequence for NGC 6342.

Nearly all bright stars that have proper motions inconsistent with cluster membership (outside the dashed circle; open symbols in Fig. 2) are brighter at a given colour than the clusterassociated ones. This is expected, since at the galactic latitude of 9.73 of our observations, most stars belonging to the disk should be in front of the cluster. We can estimate a typical distance by considering the number of stars per square arcsecond at a given distance. For an exponential distribution, this should scale as $n(d) \propto d^{2} \exp \left(-d \sin |b| / z_{0}\right)$, which reaches a maximum at $d_{\max }=2 z_{0} / \sin |b|$. Given a latitude $b=9.73$ for NGC 6342 (Harris 1996, February 2003 revision) and scale height $z_{0}=325$ pc (Reid \& Majewski 1993), 


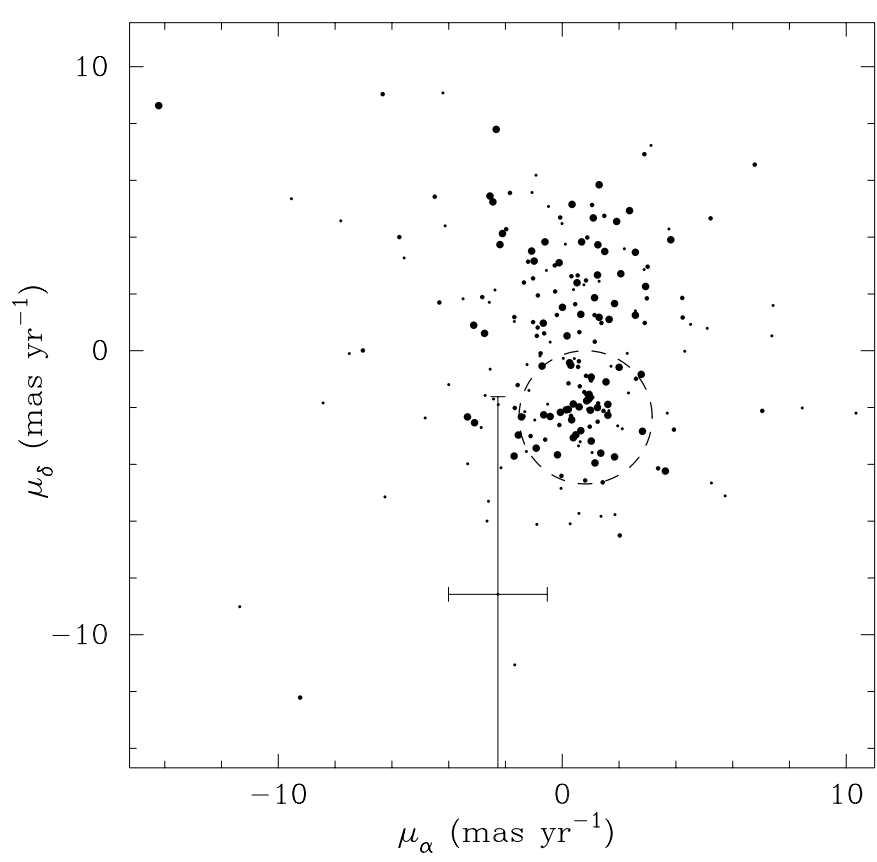

Fig. 3. Proper motion of the counterpart of PSR B1718-19 (error bar) and stars in the field. The three different sizes of the dots correspond to three brightness classes: $J<20.3,20.3 \leq J \leq 21.7$, and

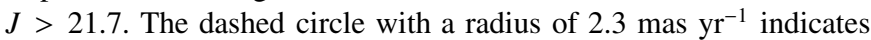
the group of stars that we associate with the cluster. The counterpart of PSR B1718-19 has a proper motion consistent with our estimate of the cluster's proper motion; the uncertainty in declination is much larger than that in right ascension due to the presence of a bright star to the south of the companion.

we obtain $d_{\max } \simeq 3.8 \mathrm{kpc}$. Since the distance of NGC 6342 is $8.6 \mathrm{kpc}$, we would thus expect stars in the galactic disks to be typically $\sim 1.7$ mag brighter than stars in NGC 6342 . This is roughly consistent with the offset seen in Fig. 2 b.

From Fig. 3, one sees that for stars with $J \gtrsim 21$, the distinction between cluster-associated and foreground stars is much less clear. This is due to the increasing uncertainty in the $J$-band position (which dominates the proper motion uncertainty). For stars with $J \lesssim 20$, the position's uncertainty is $\sim 2$ mas, while around $J \approx 21$, it has increased to $\sim 4$ mas, reaching up to $\sim 10$ mas for stars at the detection limit $(J \approx 23)$. The latter error corresponds to an error in the proper motion of 2.3 mas $\mathrm{yr}^{-1}$, equal to the radius of the dashed circle in Fig. 3.

\section{Analysis}

In this section, we estimate the companion's radius and temperature from its observed magnitudes. Since it is associated with NGC 6342, we can use the distance modulus $(m-M)_{0}=$ $14.69 \pm 0.20$ found by Heitsch \& Richtler (1999). Heitsch \& Richtler also determined the reddening, but found a rather large differential variation. Below, therefore, we first determine the reddening in our field, by fitting models to cluster stars in the colour-colour and colour-magnitude diagrammes.

Both to determine the reddening and to infer the companion's properties, we use the evolutionary tracks for low-mass stars from Baraffe et al. (1998) (hereafter, BCAH models). We use the tracks for metallicity $[\mathrm{M} / \mathrm{H}]=-0.5$, which corresponds to $[\mathrm{Fe} / \mathrm{H}] \simeq-0.77$ (Baraffe et al. 1997), close to the measured metallicity $[\mathrm{Fe} / \mathrm{H}]=-0.65$ for NGC 6342 (Harris 1996, February 2003 revision).

\subsection{Reddening}

Heitsch \& Richtler (1999) found strong evidence of differential reddening for NGC 6342, with $E_{V-I}$ varying between 0.64 and 0.74 in the core region of the cluster. At the location of PSR B1718-19, they inferred $E_{V-I}=0.70 \ldots 0.76$.

In Fig. 2a, we see that the colours of the likely cluster members are at best marginally consistent with such high reddening; a much better fit is found for a value $E_{V-I} \simeq 0.55$. To try to understand this discrepancy, we tried to determine whether the reddening varied over our smaller, $80 \times 80^{\prime \prime}$ field. We find some evidence for variation with $E_{V-I} \simeq 0.40$ at the south end and $E_{V-I} \simeq 0.75$ at the north end. Thus, it may be that the value found by Heitsch \& Richtler (1999) simply reflects a different effective centre of the region in which the reddening was determined.

Looking in detail at Fig. 2, one sees that for masses above $\sim 0.6 M_{\odot}$, the models are slightly too red in $R-I$. This could be corrected by decreasing the reddening further, but then the break in the curve at $\sim 0.6 M_{\odot}$ would happen at an $I-J$ colour that is too blue. The fact that we cannot obtain a better overall fit is most likely related to inaccuracies in the models (see Bertone et al. 2004). To take this uncertainty into account, we adopt a relatively large uncertainty in $E_{V-I}$, of $0.07 \mathrm{mag}$.

We conclude that the reddening to PSR B1718-19 is $E_{V-I}=0.55 \pm 0.07$ (or $E_{B-V}=0.40 \pm 0.05$ ). Converting reddening to extinctions in the various bands (Schlegel et al. 1998, Table 6), we find $A_{R}=1.07, A_{I}=0.78$, and $A_{J}=0.36$.

\subsection{Radius and temperature}

Below we determine the radius and temperature through a direct fit to the models. Before doing so, we make a cruder but more insightful estimate. We looked at the BCAH-models and found that, to satisfying approximation, colors depend on temperature and not on surface gravity, as long as $\Delta \log g \lesssim 1$.

From Fig. 2a, we see that the companion's colours are consistent with those of main sequence stars with a temperature of $\sim 3400 \pm 150 \mathrm{~K}$. The influence of the error in the reddening can be estimated by moving the star over the solid part of the reddening vector, which induces an error of $\sim 70 \mathrm{~K}$. Adding this in quadrature, the final estimate of the temperature is $3400 \pm 170 \mathrm{~K}$.

The companion's radius can now be estimated from Fig. $2 b$. The masses of main-sequence stars with temperatures consistent with that of the companion lie in the range $0.13 \lesssim M \lesssim$ $0.27 M_{\odot}$. Figure $2 \mathrm{~b}$ shows that these stars are fainter than the companion, which implies that the companion has a larger radius by a factor $10^{-0.2 \Delta J}$ (where $\Delta J$ is the brightness difference). From Fig. $2 \mathrm{~b}$, we estimate $\Delta J \simeq 0.3 \mathrm{mag}$ and $\Delta J \simeq$ $2.0 \mathrm{mag}$ for MS-stars of mass 0.27 and $0.13 M_{\odot}$, respectively. 
Given the main-sequence radii from the models (stars with mass $(0.13,0.25,0.30) M_{\odot}$ have $\left.\operatorname{radii}(0.15,0.26,0.30) R_{\odot}\right)$, one infers a radius of $\sim 0.31 R_{\odot}$ at the high temperature end and $0.37 R_{\odot}$ at the low end.

For a given temperature, the uncertainty in a radius thus derived has a contribution from the uncertainties in the observations and the reddening (about 0.09 mag for the $J$ band), but it is dominated by the 0.2 mag uncertainty in the distance modulus. The uncertainty in the radius for given temperature is therefore $\sim 10 \%$.

Now that we know what temperature, radius, and associated uncertainties we expect from our observations, we proceed with a fit. In order to take the uncertainty in distance and reddening into account, we define $\chi^{2}$ as

$$
\begin{aligned}
\chi^{2}= & \sum_{\mathrm{X}}\left(\frac{m_{\mathrm{X}}-A_{\mathrm{X}}^{\mathrm{var}}-\Delta m_{\mathrm{var}}+5 \log \left(R_{\mathrm{var}} / R_{\mathrm{mod}}\right)-M_{\mathrm{X}}^{\mathrm{mod}}}{\sigma_{\mathrm{X}}}\right)^{2} \\
& +\left(\frac{E_{V-I}^{\mathrm{var}}-E_{V-I}^{\mathrm{lit}}}{\sigma_{E, \mathrm{lit}}}\right)^{2}+\left(\frac{\Delta m_{\mathrm{var}}-\Delta m_{\mathrm{lit}}}{\sigma_{\Delta m, \mathrm{lit}}}\right)^{2}
\end{aligned}
$$

with $A_{\mathrm{X}}^{\mathrm{var}}=\left(A_{\mathrm{X}} / E_{V-I}\right) E_{V-I}^{\mathrm{var}}$, and $\Delta m=(m-M)_{0}$. The label "var" indicates that that part of the equation can be varied to minimize $\chi^{2}$, and the symbol $\mathrm{X}$ denotes the filter: $R, I$, or $J$. Thus, we have five measurements (three magnitudes $m_{R}$, $m_{I}$, and $m_{J}$, the distance modulus $\Delta m_{\text {lit }}$, and reddening $E_{V-I}^{\text {lit }}$ ) and four parameters (radius and temperature (which enter the model magnitudes), true distance modulus $\Delta m_{\mathrm{var}}$, and reddening $\left.E_{V-I}^{\mathrm{var}}\right)$. Using Eq. (1), we create a contourplot of $\chi^{2}$ as a function of radius and temperature in Fig. 4. Here, we have marginalized over reddening and distance modules (i.e., for every radius and temperature, reddening and distance modules are adjusted to minimize $\chi^{2}$ ). We see that the result is consistent with the crude estimate made above.

From Fig. 4, we see that the companion most likely has a radius larger than that of a main-sequence star of the same mass. The companion mass is constrained by the pulsar mass function $\left(M_{\mathrm{C}} \gtrsim 0.11 M_{\odot}\right.$ for a $1.35 M_{\odot}$ pulsar, Lyne et al. 1993). Furthermore, for given mass, the radius of the companion has to be smaller than the Roche lobe, and the temperature cannot be cooler than the Hayashi limit, where a star is completely convective. The latter limit implies that for given mass, the companion should be on or hotward of the corresponding pre-main-sequence track.

The range of allowed parameters of the candidate companion is listed in Table 3 . At the $1-\sigma$ confidence level, we observe a star with $0.11 \lesssim M \lesssim 0.27 M_{\odot}$ that is bloated compared to a main-sequence star of the same mass. Its radius lies below the Roche-limit for the mass range $M \gtrsim 0.14 M_{\odot}$. However, at the 2- $\sigma$ level we cannot exclude either a somewhat more massive main-sequence star or a lower-mass, Roche-lobe filling star (see Fig. 4). As we will see below, however, our most likely intermediate position may be most consistent with expectations from the preceding history.

\section{Discussion}

We now turn to the implications of our measurements. In doing this, we will first assume that the system formed through the

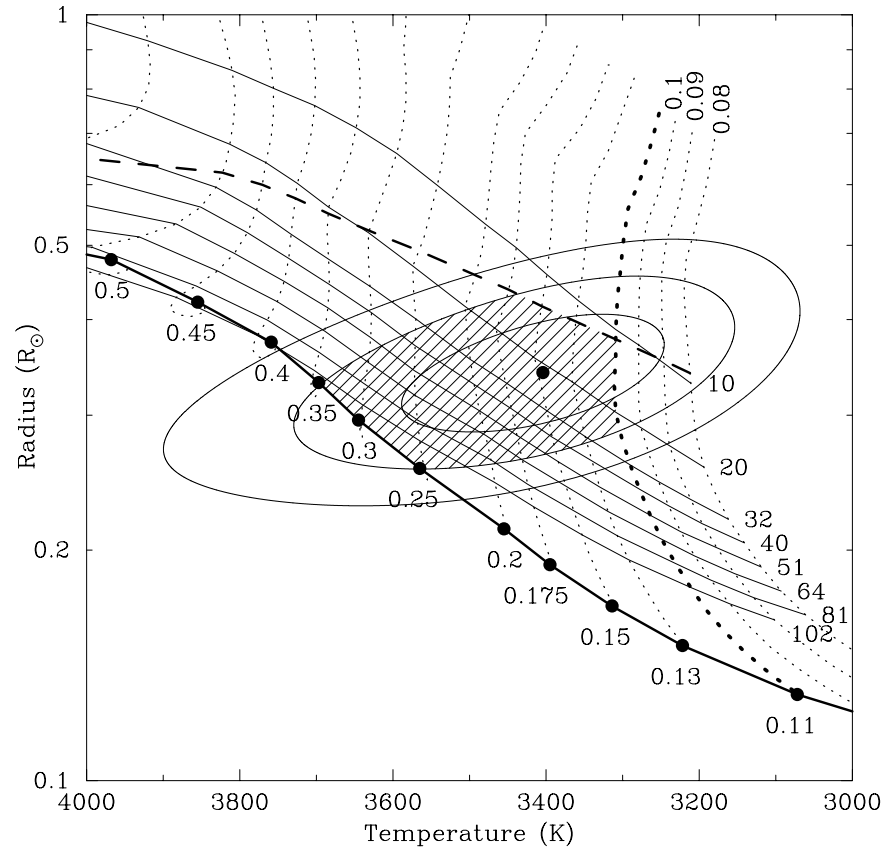

Fig. 4. Constraints on the radius and temperature of the companion of PSR B1718-19. The ellipses show the 1, 2, and 3- $\sigma$ contour levels $\left(\Delta \chi^{2}=2.30,6.17\right.$, and 11.8). The bold continuous line connecting the filled circles labeled with stellar masses (in solar units) indicates the model main sequence stars (the BCAH-models with age $10 \mathrm{Gyr}$ ). The dotted lines connect BCAH-models in a pre-main-sequence (PMS) contraction phase; the bold dotted line marks the lower limit set to the companion mass by the pulsar mass function. These PMStracks are close to the Hayashi-limit, and stars in hydrostatic equilibrium can be only on or to the left of them. The bold dashed line connects the Roche-radii for stars on the PMS-tracks. The hatched region shows the full range of allowed parameters in the $2 \sigma$ confidence region (see Table 3). Finally, the thin continuous lines are isochrones labeled with their age in Myr. These isochrones show that, within the system age of $10 \mathrm{Myr}$, the companion cannot have shrunk back to the main sequence if any significant bloating occurred that involved the whole star.

scenario of a three-body interaction in the core of the cluster. The other, in our opinion, less likely scenario of an accretioninduced collapse of a white dwarf will be discussed seperately in Sect. 4.3.

If the system formed in a three-body interaction, a bloated companion is expected, because the binary that is produced is expected to be highly eccentric, with $e \simeq 0.7$ (Phinney 1992, Sect. 5), while the system's current eccentricity is very low $(e \lesssim 0.005)$. Thus, the orbit must have circularised within the system age, which is $10 \mathrm{Myr}$ at most (Van Kerkwijk et al. 2000). The natural mechanism - tidal circularisation - transfers orbital energy to the companion, thereby bloating the star.

Below, we first estimate the expected bloating, and then look into whether tidal theory can account for the observed limit on the circularisation timescale.

\subsection{Energy balance during circularisation}

The response of a star (or planet) to the dissipation of tidal energy has been investigated by a number of authors (e.g. 
Table 3. Companion parameters, at $2 \sigma$ confidence level and at maximum probability, with the corresponding circularisation timescales.

\begin{tabular}{lccccc}
\hline \hline Position in & Temperature & Radius & Mass $^{a}$ & $\tau_{\text {circ }}[\mathrm{Z}]^{b}$ & $\tau_{\text {circ }}[\mathrm{G \& O}]^{b}$ \\
$R, T$-diagram & $(\mathrm{K})$ & $\left(R_{\odot}\right)$ & $\left(M_{\odot}\right)$ & $(\mathrm{Myr})$ & $(\mathrm{Myr})$ \\
\hline Main sequence & $3560-3700$ & $0.26-0.33$ & $0.25-0.35$ & $17-5.3$ & $(10-3.4) \times 10^{3}$ \\
Maximum probability & 3400 & 0.34 & 0.15 & 0.41 & 230 \\
Roche-lobe & $3310-3440$ & $0.38-0.43$ & $0.11-0.17$ & $0.088-0.089$ & $46-50$ \\
\hline
\end{tabular}

a The mass of the companion is estimated in Fig. 4 through interpolation of the BCAH-models.

${ }^{b}$ The circularisation timescales are computed in two prescriptions. One is described in Zahn (1989, indicated with [Z]), the other in Goodman \& Oh (1997, indicated with [G\&O]).

Ray et al. 1987; Podsiadlowski 1996; Gu et al. 2004; Ivanov \& Papaloizou 2004), but none of these studies is directly applicable to our situation. Specifically for PSR B1718-19, Verbunt (1994) showed that the tidal energy is on the order of the potential energy of the star, assuming $a_{\text {ini }} \gg a_{\mathrm{c}}$ or $e_{\text {ini }} \sim 1$ (here and below, the labels "ini" and "c" refer to those situations where the pulsar binary has just been formed and where the orbit has been circularised, respectively). With our constraints on the companion's radius and temperature, we can paint a more precise picture of the exchange of energy between orbit and stellar interior.

For our estimate, we assume that at the onset of circularisation, the companion is an ordinary main sequence star. Given the range of masses allowed for the companion (see Table 3 and Fig. 4), it will be fully convective. A measure for the timescale on which energy is distributed across the star is the convective friction time $t_{\mathrm{f}}=\left(m R^{2} / L\right)^{1 / 3}$, which varies from $0.55 \mathrm{yr}$ at the high mass end of allowed companion parameters to $0.43 \mathrm{yr}$ at the low mass end. This is much shorter than the thermal timescale in most of the star and than the circularisation timescale. We thus assume that the energy dissipated during circularisation is distributed through the entire star.

In reaction to the increase in internal energy, the star will expand until hydrostatic and approximate thermal equilibrium is regained. For a completely convective star, the total energy $E_{\star}$ is given by,

$E_{\star}=\frac{1}{2} E_{\mathrm{pot}} \simeq-\frac{3 G m^{2}}{7 R}$,

where we used the Virial theorem and assumed the star's potential energy $E_{\text {pot }}$ was roughly that of a $n=1.5$ polytrope (Chandrasekhar 1939, Eq. (90)), as appropriate for a completely convective star. The symbols $m$ and $R$ denote the companion's mass and radius, respectively.

Next, we use the fact that orbital angular momentum should be roughly conserved in the circularisation process (the companion's rotational angular momentum is much smaller than the orbital angular momentum). This implies $a_{\text {ini }}\left(1-e_{\text {ini }}^{2}\right)=$ $a_{\mathrm{c}}\left(1-e_{\mathrm{c}}^{2}\right) \approx a_{\mathrm{c}}$, and hence,

$E_{\text {orbit,ini }}=\frac{G m M}{2 a_{\text {ini }}} \approx E_{\text {orbit, } \mathrm{c}}\left(1-e_{\text {ini }}^{2}\right)$,

where $M$ is the mass of the neutron star, for which we take $1.35 M_{\odot}$ (Thorsett \& Chakrabarty 1999), and $a$ the semimajor axis, which can be computed for given companion mass using the observed period of 22314.83 s (Lyne et al. 1993). Combining Eqs. (2) and (3), we can write the exchange of orbital energy and total energy of the companion, $\Delta E_{\text {orbit }}=$ $\Delta E_{\star}$, as:

$\frac{G m M}{2 a_{\mathrm{c}}} e_{\mathrm{ini}}^{2}=-\frac{3 G m^{2}}{7}\left[\frac{1}{R_{\mathrm{c}}}-\frac{1}{R_{\mathrm{MS}}}\right]$,

where we assume that the initial radius $R_{\text {ini }}$ is that of a mainsequence star, $R_{\mathrm{MS}}$. Here, we neglected the contribution from the companion's rotational energy, since this is much smaller than the change in orbital energy.

For a given companion mass, we can use Eq. (4) to calculate the initial eccentricity that induces bloating of the main sequence star up to a given radius. In this way, we convert the $1 \sigma$ and $2 \sigma$ contours in the radius-temperature diagramme (Fig. 4) into corresponding contours in an eccentricity-mass diagramme (Fig. 5), where we determine the mass by assuming the star is presently on a cooling track.

From Eq. (4) we can also calculate the critical initial eccentricity, which is the eccentricity for which the main sequence star is bloated exactly to the Roche limit. For a rough estimate, we approximate the semi-major axis, which is only weakly dependent on $m$, by $a_{\mathrm{c}} \approx 2 R_{\odot}$, and the Roche-limit by $R_{L} \approx 0.46 a(m /(m+M))^{1 / 3}$ (Paczyński 1971). Applying the mass-radius relation $R_{\mathrm{MS}} / R_{\odot} \approx m_{\mathrm{MS}} / M_{\odot}$, we obtain

$e_{\mathrm{crit}}^{2} \simeq 1.270-0.553\left(\frac{m}{0.2 M_{\odot}}\right)^{2 / 3}$.

In Fig. 5, one sees that the approximation agrees rather well with the values determined directly from the model; the differences are largely due to the inaccuracy in the assumed massradius relation.

In theory, the companion could fill its Roche lobe not only at the end of circularisation, when the companion's radius is maximal, but also at earlier stages when the radius is smaller but the orbital separation at periastron is smaller as well. To find the radius at any value of $e$, we solve the differential equation,

$\frac{\mathrm{d} E_{\star}(R)}{\mathrm{d} e}=-\frac{\mathrm{d} E_{\text {orbit }}(e)}{\mathrm{d} e} \Rightarrow-\frac{3 G m^{2}}{7} \frac{\mathrm{d}(1 / R)}{\mathrm{d} e}=-\frac{G m M}{a_{\mathrm{c}}} e,(6)$

where we again assumed conservation of orbital angular momentum. We find

$R(e)=\frac{R_{\mathrm{MS}}}{1+\left(7 M R_{\mathrm{MS}}\right) /\left(6 m a_{\mathrm{c}}\right)\left(e^{2}-e_{\mathrm{ini}}^{2}\right)}$. 


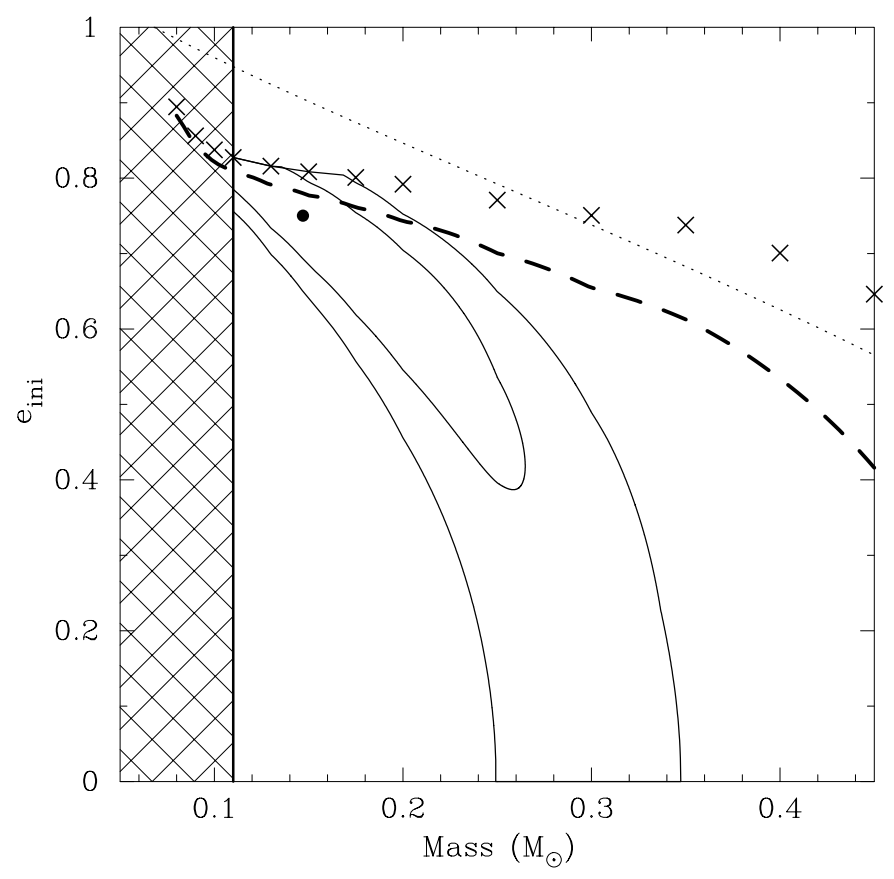

Fig. 5. Initial eccentricity $e_{\text {ini }}$ for which the change in orbital energy $\Delta E_{\text {orbit }}$ during circularisation equals the excess energy $E_{\star, \mathrm{obs}}-$ $E_{\star, M S}$ of the present companion relative to a main-sequence star (see Eq. (4)). Shown are the initial eccentricities that can account for the bloating observed at the best-fit point as well as at the 1 and 2- $\sigma$ contours from Fig. 4. The hatched region indicates the lower limit to the companion mass of $0.11 M_{\odot}$. The dotted line shows the estimate from Eq. (5) for the critical initial eccentricity at which the star would be bloated to the Roche lobe. The crosses mark the critical eccentricities found from a more careful calculation; the main difference is due to the use of the more accurate main-sequence radii from the BCAH-models. The bold dashed line indicates the critical eccentricity above which Roche lobe overflow occurs at any time during the circularisation process (see text).

Requiring that this radius is smaller than the Roche limit (using the approximation of Eggleton 1983) throughout the circularisation process, we can compute an upper limit to the initial eccentricity above which Roche lobe overflow occurs. This upper limit is also shown in Fig. 5.

Above, we neglected any energy loss during or after circularisation. From the isochrones in Fig. 4 we conclude that, within the system age of $10 \mathrm{Myr}$, the star cannot have shrunk back significantly due to radiative losses. A contraction timescale $\left(E_{\star} / L\right)$ substantially longer than the tidal bloating timescale $\left(E_{\star} / L_{\text {tide }}\right)$ is indeed expected, since the star's luminosity of $\sim 0.014 L_{\odot}$ is substantially smaller than the tidal luminosity $L_{\text {tide }} \gtrsim\left(e_{\text {ini }}^{2} G m M / 2 a_{\mathrm{c}}\right) / \tau=0.08 L_{\odot}$. This leaves only one other potential flaw in our approach: whether the energy is dissipated throughout the whole star. This dissipation could be confined to a thin layer (see the discussion in Sect. 5.2 of Van Kerkwijk et al. 2000), and thereby turn the energy-transport beneath the layer radiative. This would require a much more sophisticated treatment, if only because of the implications for the dissipation mechanism itself.

In summary, if our assumption that the dissipation energy is distributed throughout the whole star holds, Fig. 5 shows that our measurement of the companion's temperature and radius is in good agreement with the initial eccentricity of $\sim 0.7$ expected from the formation model.

\subsection{Circularisation timescale}

Regardless of the dissipation mechanism, tidal circularisation is expected to produce a decay of the eccentricity that is exponential. To reduce the eccentricity from the expected initial eccentricity of $\sim 0.7$ to the tiny currently observed value of $\lesssim 0.005$ therefore requires at least four circularisation timescales. Since the system is likely to be in its present state for at most $10 \mathrm{Myr}$ (Van Kerkwijk et al. 2000), the circularisation timescale must have been $\lesssim 2.5 \mathrm{Myr}$.

A star of a mass in the observed range is expected to be fully convective. The dominant dissipation mechanism in a convective star is thought to be the friction between the convective motions and the tidal flow, produced by turbulent viscosity (see, e.g., Zahn 1989). This mechanism becomes less efficient if - like in our case - the convective turnover timescale is longer than the tidal period, but it is not clear to what extent. We use two prescriptions for the effect of the timescale mismatch: Zahn (1989), in which the efficiency is assumed to decrease linearly with the ratio convective-to-orbital timescale, and Goodman \& Oh (1997), in which the efficiency decreases almost quadratically. For details on these calculations, see Van Kerkwijk et al. (2000). We should stress that at present it is not clear that either prescription is reliable; see Goodman \& Oh (1997) for a discussion.

The resulting theoretical circularisation timescales are listed in Table 3. It is clear that in Goodman \& Oh (1997)'s prescription the derived timescales are too long by at least an order of magnitude, whereas in Zahn (1989)'s prescription a timescale is derived that is consistent with the upper limit cited above. However, this is only true if the companion is bloated. This reinforces the conclusion derived from studies of the circularisation of main-sequence binaries that our current theories underestimate the efficiency of tidal energy dissipation (see Meibom \& Mathieu 2004 for a recent summary of the observations).

\subsection{The AIC-scenario}

The main alternative formation scenario assumes that the system formed through the accretion-induced collapse (AIC) of a white dwarf. In this scenario, the companion was filling its Roche-lobe at the time of collapse. The kick that is required to bring the system to its present location far outside the cluster core, increases the orbital separation by only a factor of $\sim 1.25$. Therefore, the companion should still be close to filling its Roche-lobe. The only possible way to explain the observed faint companion, is that the companion was bloated before collapse, by irradiation from the accreting white dwarf (as suggested by Wijers \& Paczynski 1993). As it is not clear whether this is possible by irradiating only one side of the star (King et al. 1996), we regard the AIC-scenario as the less likely formation model for this system. 
In this scenario, an initial eccentricity of $\sim 0.2$ is expected (Van Kerkwijk et al. 2000). Therefore, the circularisation timescale required to explain the current circular orbit is roughly the same as for a scenario in which the system is formed by a triple interaction ( $\$ 2.5 \mathrm{Myr}$ ). Even taking into account that the companion will be close to Roche-lobe filling, one sees from Table 3 that the circularisation timescale can be matched theoretically only using the prescription of Zahn (1989). Thus, our conclusions in Sect. 4.2 remain unchanged.

It is unclear in what precise state the companion is expected to be immediately after collapse. For a crude estimate, we assume that at the moment of collapse, the companion had a radius equal to the Roche limit, and use Eq. (4) to compute the critical eccentricity, substituting $R_{\mathrm{c}}=R_{\mathrm{L}}=(1 / 1.25) R_{\text {ini. }}$. This yields,

$e_{\text {crit }}^{2}=0.1383\left(\frac{m}{0.2 M_{\odot}}\right)$,

where we used the same approximations for the semi-major axis and the Roche limit as in the derivation of Eq. (5). This is consistent with our measurement of a companion that is bloated to a radius smaller than the Roche limit, and thus does not add further constraints on which formation scenario is the correct one.

\section{Conclusion}

We have used VLT observations to measure the temperature and radius of the likely companion of PSR B1718-19. In conjunction with the companion mass inferred from radius and temperature employing pre-main-sequence cooling tracks, these point to a companion that is bloated, but does not fill its Roche-lobe.

We have shown that a bloated companion is expected in the context of the scenario in which the system formed in a threebody interaction in the cluster core less than $10 \mathrm{Myr}$ ago. With the eccentricity that is expected to arise from such an event, tidal circularisation can supply the energy needed for a main sequence star of the observed mass to expand to the observed radius.

We tried to compare the observed upper limit on the circularisation time scale with theory, but were hampered by the uncertainty in the extent to which the circularisation efficiency is suppressed by the orbital period being shorter than the convective turnover time. The system constitutes an observational constraint on any theoretical account of tidal circularisation.

The constraints on the properties of the companion could be improved by even deeper observations, as these would allow comparison of the companion with main sequence stars of the same color, thus removing the uncertainty in distance and reddening. At this point, however, it seems more urgent to establish a satisfying theoretical account of the tidal dissipation mechanism that is dominant in a fully convective star.

Acknowledgements. We thank Ferdi Hulleman and Cees Bassa for help with the photometry, and Cees Bassa, Peter Stetson, and
Andy Dolphin for useful discussions on HST photometry. This research made extensive use of the ADS, SIMBAD, and Vizier data bases.

\section{References}

Amico, P., Cuby, J. G., Devillard, N., Jung, Y., \& Lidman, C. 2001, ISAAC-SW Data Reduction Guide 1.4

Baggett, S., et al. 2002, in HST WFPC2 Data Handbook, ed. B. Mobasher, et al.

Baraffe, I., Chabrier, G., Allard, F., \& Hauschildt, P. H. 1997, A\&A, 327, 1054

Baraffe, I., Chabrier, G., Allard, F., \& Hauschildt, P. H. 1998, A\&A, 337, 403

Bertone, E., Buzzoni, A., Chavez, M., \& Rodriguez-Merino, L. H. 2004, AJ, in press [arXiv: astro-ph/0406215]

Burderi, L., \& King, A. R. 1994, ApJ, 430, L57

Chandrasekhar, S. 1939, An introduction to the study of stellar structure (Chicago: The University of Chicago press)

Devillard, N. 1997, The Messenger, 87, 19

Dolphin, A. E. 2000, PASP, 112, 1383

Eggleton, P. P. 1983, ApJ, 268, 368

Ergma, E. 1993, A\&A, 273, L38

Ergma, E., Sarna, M. J., \& Giersz, M. 1996, A\&A, 307, 768

Goodman, J., \& Oh, S. P. 1997, ApJ, 486, 403

Gu, P., Bodenheimer, P. H., \& Lin, D. N. C. 2004, ApJ, 608, 1076

Harris, W. E. 1996, AJ, 112, 1487

Heitsch, F., \& Richtler, T. 1999, A\&A, 347, 455

Ivanov, P. B., \& Papaloizou, J. C. B. 2004, MNRAS, 347, 437

King, A. R., Frank, J., Kolb, U., \& Ritter, H. 1996, ApJ, 467, 761

Lyne, A. G., Biggs, J. D., Harrison, P. A., \& Bailes, M. 1993, Nature, 361,47

Mathieu, R. D., Meibom, S., \& Dolan, C. J. 2004, ApJ, 602, L121

Meibom, S., \& Mathieu, R. D. 2004, ApJ, in press

Moorwood, A., Cuby, J.-G., Biereichel, P., et al. 1998, The Messenger, 94, 7

Paczyński, B. 1971, ARA\&A, 9, 183

Persson, S. E., Murphy, D. C., Krzeminski, W., Roth, M., \& Rieke, M. J. 1998, AJ, 116, 2475

Phinney, E. S. 1992, Phil. Trans. R. Soc. London, A, 341, 39

Podsiadlowski, P. 1996, MNRAS, 279, 1104

Ray, A., Kembhavi, A. K., \& Antia, H. M. 1987, A\&A, 184, 164

Reid, N., \& Majewski, S. R. 1993, ApJ, 409, 635

Schlegel, D. J., Finkbeiner, D. P., \& Davis, M. 1998, ApJ, 500, 525

Seifert, W., Appenzeller, I., Fuertig, W., et al. 2000, in Proc. SPIE, ed. M. Iye, \& A. F. Moorwood, 4008, 96

Stetson, P. B. 1987, PASP, 99, 191

Stetson, P. B. 2000, PASP, 112, 925

Thorsett, S. E., \& Chakrabarty, D. 1999, ApJ, 512, 288

Van Kerkwijk, M. H., Kaspi, V. M., Klemola, A. R., et al. 2000, ApJ, 529,428

Van Kerkwijk, M. H., \& Kulkarni, S. R. 2001, A\&A, 378, 986

Verbunt, F. 1994, A\&A, 285, L21

Verbunt, F., \& Phinney, E. S. 1995, A\&A, 296, 709

Wijers, R. A. M. J., \& Paczynski, B. 1993, ApJ, 415, L115

Wu, Y. 2003, in ASP Conf. Ser., 294, 213

Zahn, J.-P. 1989, A\&A, 220, 112

Zwitter, T. 1993, MNRAS, 264, L3 